\title{
Pattern of comorbidities and 1-year mortality in elderly patients with COPD hospitalized in internal medicine wards: data from the RePoSI Registry
}

\author{
Christiano Argano ${ }^{1}$ - Nicola Scichilone ${ }^{2}$. Giuseppe Natoli ${ }^{1}$. Alessandro Nobili ${ }^{3}$. Gino Roberto Corazza ${ }^{4}$

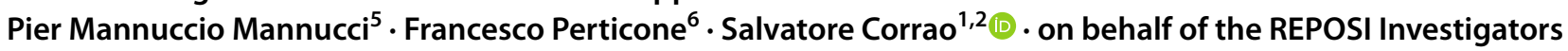

Received: 18 April 2020 / Accepted: 16 June 2020

(c) Società Italiana di Medicina Interna (SIMI) 2020

\begin{abstract}
Currently, chronic obstructive pulmonary disease (COPD) represents the fourth cause of death worldwide with significant economic burden. Comorbidities increase in number and severity with age and are identified as important determinants that influence the prognosis. In this observational study, we retrospectively analyzed data collected from the RePoSI register. We aimed to investigate comorbidities and outcomes in a cohort of hospitalized elderly patients with the clinical diagnosis of COPD. Socio-demographic, clinical characteristics and laboratory findings were considered. The association between variables and in-hospital, 3-month and 1-year follow-up were analyzed. Among 4696 in-patients, 932 (19.8\%) had a diagnosis of COPD. Patients with COPD had more hospitalization, a significant overt cognitive impairment, a clinically significant disability and more depression in comparison with non-COPD subjects. COPD patients took more drugs, both at admission, in-hospital stay, discharge and 3-month and 1-year follow-up. 14 comorbidities were more frequent in COPD patients. Cerebrovascular disease was an independent predictor of in-hospital mortality. At 3-month follow-up, male sex and hepatic cirrhosis were independently associated with mortality. ICS-LABA therapy was predictor of mortality at in-hospital, 3-month and 1-year follow-up. This analysis showed the severity of impact of COPD and its comorbidities in the real life of internal medicine and geriatric wards.
\end{abstract}

Keywords COPD, comorbidities, elderly, internal medicine, in-hospital mortality $\cdot 3$-month mortality $\cdot 1$-year mortality

\section{Introduction}

The members of the group of RePoSI Registry mentioned in Acknowlegements section.

Salvatore Corrao

salvatore.corrao@unipa.it

1 Internal Medicine Department, UOC Medicina Interna iGR, National Relevance Hospital Trust, ARNAS Civico, Di Cristina e Benfratelli, Piazza Nicola Leotta, 2, 90127 Palermo, Italy

2 Dipartimento di Promozione della Salute, Materno Infantile, Medicina Interna e Specialistica di Eccellenza "G. D’Alessandro, University of Palermo, Palermo, Italy
Chronic obstructive pulmonary disease (COPD) represents an important leading cause of morbidity and mortality with high economic and social costs: according to the WHO, COPD is the fourth most common cause of death worldwide, and it is estimated to be the third by 2020; furthermore, the global burden of COPD is expected to increase in the

3 Department of Neuroscience, IRCCS Istituto di Ricerche Farmacologiche Mario Negri, Milan, Italy

4 Department of Internal Medicine, University of Pavia and San Matteo Hospital, Pavia, Italy

5 Scientific Direction, IRCCS Foundation Maggiore Hospital Policlinico, Milan, Italy

6 Department of Medical and Surgical Sciences, University Magna Graecia of Catanzaro, Catanzaro, Italy 
coming years, due to the prevalence of smoking and aging of the world population [1].

Comorbidities are an essential component of COPD burden. Some of these are related to aging, others may have the same underlying mechanisms (e.g. systemic inflammation) or share common risk factors (e.g. smoking exposure), but all of them are able to afflict prognosis [2]. Some comorbidities occur more frequently in COPD patients, independently from pulmonary severity disease [3]. They increase in number and severity with age and have a major impact on the patient's quality of life, hospitalization and mortality [4]. In this sense, Divo and colleagues identified twelve comorbidities associated with increased mortality [5]. However, recommendations on management of respiratory diseases are based on evidence from studies with restrictive inclusion criteria or no representative enrollment $[6,7]$, thus not accounting for complicating effects from coexisting conditions and treatments. Therefore, their management and prevention might provide benefit in reducing the global cost load especially since international recommendations on COPD management do not systematically include the evaluation of comorbid conditions in the diagnostic approach or in the treatment decisions of the disease, thus focusing on isolated lung impairment rather than multimorbidity. Given this background, the aim of this study was to assess comorbidities and outcomes in a cohort of elderly patients with the clinical diagnosis of COPD, hospitalized in Internal Medicine and Geriatric Wards participating to the RePoSI (REgistro per lo studio delle POlipatologie e politerapie SImi) registry study.

\section{Methods}

\section{Data collection and study population}

Retrospectively, we analyzed the collected data within the frame of the RePoSI project in the recruitment weeks of 2010, 2012, 2014 and 2016. RePoSI is an independent and collaborative register, organized by the Italian Society of Internal Medicine (SIMI) and the Mario Negri Institute for Pharmacological Research. It involved the creation of a network of internal medicine and geriatric wards that collected information about polytherapy on elderly patients, affected by multiple diseases. Patients were eligible for RePoSI if: (1) they were admitted to one of the participating internal medicine wards during the 4 index weeks chosen for recruitment (one in February, one in June, one in September, and one in December); (2) their age was 65 years or older; (3) they gave informed consent. Each ward had to enroll at least ten consecutive eligible patients during each index week recording data on socio-demographic details, the main reason for admission and comorbidities, diagnoses, treatment (including all drugs taken at hospital admission and recommended at discharge), clinical events during hospitalization and outcome. During those weeks, all participating centers had to complete the registration of all patients admitted, indicating those who were consecutively enrolled. For patients who were excluded, the reason had to be given. Also, data on mortality or any new hospitalization were collected, with a telephone interview performed by a physician to the patient or his/her relatives, 3 -and 12 months after hospital discharge. Then, a final database was created and checked by the Mario Negri Institute for Pharmacological Research. The project's design is accessible at the related website [8]. Subjects were referred as having COPD if a diagnosis of the disease was reported in previous medical charts, or whether the diagnosis was posed at admission, as judged by the clinician. Given the nature of the study, the spirometric assessment was judged not to be a pre-requisite to confirm the diagnosis.

\section{Socio-demographic and clinical characteristics}

Socio-demographic variables such as age classes, marital status, living arrangement and need for assistance in daily living, were considered along with laboratory findings in patients with COPD compared to the ones without it. The following clinical characteristics were evaluated: respiratory and non-respiratory disease distribution at hospital admission (according to International Classification of DiseasesNinth Revision); cognitive status and mood disorders (by the Short-Blessed-Test [SBT] [9] and the Geriatric-DepressionScale [GDS] [10],respectively; performance in activities of daily living at hospital admission (measured by means of the Barthel Index [BI] [11]; severity and comorbidity index(assessed by the Cumulative-Illness-Rating-Scale CIRS-s and CIRS-c, respectively) [12], glomerular filtration rate (using the Chronic Kidney Disease Epidemiology Collaboration-formula [13]), length of hospital stay, drugs prescriptions (at admission, discharge, at 3 and 12 months follow-up), destination at discharge, in-hospital and 3-month and 1-year mortality rate. The association between variables and in-hospital, 3-month and 1-year mortality was analyzed.

\section{Statistical analysis}

Quantitative variables were summarized as mean (95\% confidence intervals), and categorical variables as percentage. Patients with significant disability were selected according to a BI score of $\leq 40$. Fisher's exact-test for contingency tables, z test and non-parametric Mann-Whitney $U$ test were used when appropriate. A multivariate logistic analysis was used to assess the relationship between variables and inhospital, 3-month and 1-year follow-up mortality. Variables 
were chosen according to the Hosmer-Lemeshow methodology [14]. After univariate analysis, only variables with a $p<0.20$ were included in the final model; then, through a backward process, variables were excluded until a significance level of $p<0.20$ was reached for each variable. The application of Hosmer-Lemeshow test is a measure of how well the model fits the data without any choice of variables by researcher to put into the multivariate model. A two-tailed $p<0.05$ was considered statistically significant. Stata Statistical Software2016, Release14 (Stata-Corp,
College-Station, TX-USA) was used for database management and all the analyses.

\section{Results}

During the recruitment period, 4696 out of 4825 inpatients were eligible for this analysis (129 patients had missed variables); 932 (19.8\%) presented with a diagnosis of COPD. Among them, $61 \%$ were male with a mean age of
Table 1 Socio-demographic characteristics and modifiable risk factors of the REPOSI elderly population according to Chronic Obstructive Pulmonary Disease (COPD) categorization

\begin{tabular}{|c|c|c|c|}
\hline Variables & Inpatient with COPD & Inpatient without COPD & $p$ \\
\hline$N^{\circ}$ of subjects & 932 & 3764 & - \\
\hline $\operatorname{Men}(\%)$ & 61.0 & 46.1 & $<0.0001$ \\
\hline $\mathrm{Age}^{\mathrm{a}}$ & $80.1(79.6-80.5)$ & $79.4(79.1-79.6)$ & 0.0064 \\
\hline Marital status (\%) & & & 0.724 \\
\hline Married & 52.4 & 54.2 & \\
\hline Widow & 38.6 & 36.4 & \\
\hline Separated & 1.4 & 1.3 & \\
\hline Divorced & 1.4 & 1.4 & \\
\hline Living arrangement $(\%)$ & & & 0.444 \\
\hline Alone & 23.5 & 22.7 & \\
\hline Spouse & 43.9 & 45.2 & \\
\hline Sons & 15.6 & 15.7 & \\
\hline Spouse and sons & 7.2 & 6.9 & \\
\hline Other & 9.4 & 8.5 & \\
\hline Previously Institutionalized (\%) & 6.0 & 5.6 & 0.5977 \\
\hline Previously Hospitalized (\%) & 46.2 & 35.0 & 0.0002 \\
\hline Caregiver $(\%)$ & 58.6 & 51.1 & $<0.0001$ \\
\hline Spouse $(\%)$ & 37.6 & 32.0 & 0.091 \\
\hline Brother/Sister (\%) & 3.0 & 3.4 & \\
\hline Son/Daughter (\%) & 42.9 & 47.9 & \\
\hline Son/Daughter in law (\%) & 0.6 & 1.5 & \\
\hline Grandson $(\%)$ & 3.6 & 3.7 & \\
\hline Other $(\%)$ & 12.4 & 11.4 & \\
\hline Never Smoked (\%) & 32.1 & 60.0 & $<0.0001$ \\
\hline ex-Smoker (\%) & 52.8 & 33.0 & \\
\hline Smoker $(\%)$ & 15.0 & 7.0 & \\
\hline Never Alcohol (\%) & 47.8 & 58.8 & $<0.0001$ \\
\hline Alcohol (\%) & 38.9 & 26.0 & \\
\hline ex-Alcohol (\%) & 4.8 & 6.4 & \\
\hline Casual Drinking (\%) & 8.5 & 8.8 & \\
\hline $\mathrm{BMI}^{\mathrm{a}}$ & $26.26(25.87-26.65)$ & $25.84(25.67-26.01)$ & 0.1332 \\
\hline Underweight patients (\%) & 4.2 & 3.9 & 0.6913 \\
\hline Optimal weight patients (\%) & 39.6 & 41.5 & 0.3021 \\
\hline Overweight patients (\%) & 34.1 & 35.6 & 0.4176 \\
\hline Class I obesity (\%) & 13.6 & 12.5 & 0.3766 \\
\hline Class II obesity (\%) & 3.7 & 3.0 & 0.2967 \\
\hline Class III obesity (\%) & 2.4 & 1.3 & 0.0136 \\
\hline
\end{tabular}

${ }^{a}$ Data are reported as mean (95\% confidence interval) 
80 years. Table 1 shows the demographic characteristics and modifiable risk factors of the two study groups.

Interestingly, almost half of the COPD in-patients had history of previous hospitalizations compared to only onethird of non-COPD inpatients. A significantly higher proportion of COPD subjects also showed history of alcohol consumption and were more often morbidly obese.

In-patients with COPD had a significantly higher cumulative illness rating scale for the evaluation of severity and comorbidity index ( $p<0.0001$ for both comparisons). As shown in Table 2, significant overt cognitive impairment was documented in almost half of in-patients with COPD, while a quarter needed positioning of urinary catheter. In-patients with clinically significant disability $(\mathrm{BI} \leq 40)$ were $16.8 \%$ in comparison with individuals without COPD $(13.9 \%, p=0.0291)$. Moreover, GDS was shown to be more frequently abnormal (mean-score equal to 1.49). In addition, $21.2 \%$ had a probable depression (GDS $>2$ ) as opposed to non-COPD individuals. COPD patients took more drugs than those without COPD, both at admission, at in-hospital stay, at discharge and at 3-and 1-year followup (Table 2).

Overall, disease distribution showed that arterial hypertension, ischemic heart disease, atrial fibrillation, heart failure, chronic renal failure, peripheral artery disease, overt hypertensive heart disease, anemia, rheumatic diseases, prostatic hypertrophy, osteoporosis, pneumonia,

Table 2 Laboratory and clinical characteristics of the REPOSI population at hospital admission according to Chronic Obstructive Pulmonary Disease (COPD) categorization

\begin{tabular}{|c|c|c|c|}
\hline Variables & Inpatient with COPD & Inpatient without COPD & $p$ \\
\hline Systolic blood pressure $(\mathrm{mm} \mathrm{Hg})^{\mathrm{a}}$ & $130.0(128.7-131.3)$ & $132.3(131.6-133.1)$ & 0.0089 \\
\hline Diastolic blood pressure $(\mathrm{mm} \mathrm{Hg})^{\mathrm{a}}$ & $73.4(72.7-74.1)$ & $73.6(73.2-73.9)$ & 0.6558 \\
\hline Heart rate $(\mathrm{bpm})^{\mathrm{a}}$ & $80.0(78.9-81.0)$ & $78.8(78.2-79.3)$ & 0.0108 \\
\hline Body temperature $\left({ }^{\circ} \mathrm{C}\right)^{\mathrm{a}}$ & $36.89(36.78-37.01)$ & $37.99(35.94-40.04)$ & 0.0106 \\
\hline Fasting glucose $(\mathrm{mg} / \mathrm{dL})^{\mathrm{a}}$ & $129.9(126.0-133.8)$ & $126.8(124.7-128.8)$ & 0.0247 \\
\hline Creatinine $(\mathrm{mg} / \mathrm{dL})^{\mathrm{a}}$ & $1.28(1.22-1.34)$ & $1.26(1.23-1.29)$ & 0.0112 \\
\hline $\mathrm{GFR}(\mathrm{ml} / \mathrm{min})^{\mathrm{a}}$ & $58.9(57.4-60.5)$ & $59.4(58.6-60.2)$ & 0.3902 \\
\hline Mild decrease in GFR(ml/min) & 37.7 & 41.2 & 0.0519 \\
\hline Moderate decrease in $\mathrm{GFR}(\mathrm{ml} / \mathrm{min})$ & 39.2 & 35.5 & 0.0340 \\
\hline Severe decrease in GFR(ml/min) & 10.2 & 10.1 & 0.9257 \\
\hline Kidney Failure & 2.6 & 3.7 & 0.0907 \\
\hline Hemoglobin $(\mathrm{mg} / \mathrm{dL})^{\mathrm{a}}$ & $12.09(11.94-12.23)$ & $11.76(11.68-11.83)$ & 0.0002 \\
\hline Leucocytes (cells per microliter) $\left(\times 10^{3} / \mathrm{uL}\right)^{\mathrm{a}}$ & $9.94(9.40-10.47)$ & $9.83(9.19-10.46)$ & $<0.0001$ \\
\hline Platelets (cells per microliter) $\left(\times 10^{3} / \mathrm{uL}\right)^{\mathrm{a}}$ & $232.33(226.03-238.63)$ & $229.94(226.26-233.63)$ & 0.1314 \\
\hline Cholesterol $(\mathrm{mg} / \mathrm{dL})^{\mathrm{a}}$ & $160.6(157.3-163.9)$ & $159.4(157.6-161.1)$ & 0.4738 \\
\hline Short Blessed Test score ${ }^{a}$ & $9.6(9.1-10.2)$ & $9.0(8.7-9.3)$ & 0.0169 \\
\hline $\begin{array}{l}\text { Overt Cognitive impairment (Short Blessed Test } \\
\text { score } \geq 10)(\%)\end{array}$ & 39.4 & 35.1 & 0.0202 \\
\hline Need for urinary catheter $(\%)$ & 25.5 & 21.7 & 0.0155 \\
\hline Barthel index score ${ }^{a}$ & $73.8(71.8-75.7)$ & $78.5(77.5-79.4)$ & $<0.0001$ \\
\hline $\begin{array}{l}\text { Clinically significant disability } \\
\text { (Barthel index } \leq 40)(\%)\end{array}$ & 16.8 & 13,9 & 0.0291 \\
\hline Geriatric Depression Scale score $^{a}$ & $1.47(1.39-1.56)$ & $1.37(1.33-1.41)$ & 0.0380 \\
\hline $\begin{array}{l}\text { Probable Depression } \\
\text { (Geriatric Depression Scale score }>2)(\%)\end{array}$ & 21.2 & 17.7 & 0.0222 \\
\hline$N^{\circ}$ of drugs at hospital admission ${ }^{\mathrm{a}}$ & $6.7(6.5-6.9)$ & $5.5(5.4-5.6)$ & $<0.0001$ \\
\hline$N^{\circ}$ of in-hospital drugs ${ }^{\mathrm{a}}$ & $8.7(8.4-9.1)$ & $7.7(7.5-7.8)$ & $<0.0001$ \\
\hline$N^{\circ}$ of drugs at hospital discharge ${ }^{\mathrm{a}}$ & $8.4(8.1-8.7)$ & $7.5(7.4-7.6)$ & $<0.0001$ \\
\hline$N^{\circ}$ of drugs at follow up 3 months ${ }^{\mathrm{a}}$ & $7.4(7.1-7.7)$ & $6.4(6.2-6.5)$ & $<0.0001$ \\
\hline$N^{\circ}$ of drugs at follow up 1 year ${ }^{\mathrm{a}}$ & $7.3(6.6-7.9)$ & $6.2(5.9-6.5)$ & 0.0020 \\
\hline Severity index (by CIRS) ${ }^{a}$ & $1.79(1.77-1.82)$ & $1.64(1.63-1.65)$ & $<0.0001$ \\
\hline Comorbidity index (by CIRS) ${ }^{a}$ & $3.66(3.54-3.79)$ & $2.90(2.84-2.96)$ & $<0.0001$ \\
\hline
\end{tabular}

$B M I$ Body Mass Index, CIRS cumulative illness rating scale

${ }^{a}$ Data are reported as mean (95\% confidence interval) 
Table 3 The most frequent clinical diagnoses (as percentage) in the REPOSI population according to Chronic Obstructive Pulmonary Disease (COPD) categorization (the table only shows the diagnoses which frequency was more than $5 \%$ at least in one group)
Table 4 Length of hospital stay, destination at hospital discharge, in-hospital and at follow-up mortality of the whole REPOSI population according to Chronic Obstructive Pulmonary Disease (COPD) categorization

\begin{tabular}{|c|c|c|c|}
\hline Variables & $\begin{array}{l}\text { Inpatient with } \\
\text { COPD }(\%)\end{array}$ & $\begin{array}{l}\text { Inpatient without COPD } \\
(\%)\end{array}$ & $p$ \\
\hline Arterial hypertension & 65.8 & 57.5 & $<0,0001$ \\
\hline Diabetes & 31.9 & 28.7 & 0.0567 \\
\hline Ischemic heart disease & 31.1 & 20.8 & $<0,0001$ \\
\hline Atrial fibrillation & 29.2 & 23.5 & 0.0003 \\
\hline Heart Failure & 29.4 & 17.6 & $<0,0001$ \\
\hline Chronic renal failure & 27.4 & 18.5 & $<0,0001$ \\
\hline Peripheral artery disease & 19.5 & 13.5 & $<0,0001$ \\
\hline Cancer & 19.1 & 18.9 & 0.8904 \\
\hline Overt hypertensive heart disease & 17.4 & 11.9 & $<0,0001$ \\
\hline Anemia & 17.1 & 21.3 & 0.0043 \\
\hline Rheumatic diseases & 16.5 & 12.2 & 0.0045 \\
\hline Prostatic hypertrophy & 16.0 & 10.2 & $<0,0001$ \\
\hline Gastritis & 14.5 & 12.9 & 0.1965 \\
\hline Dementia & 14.2 & 15.1 & 0.5046 \\
\hline Arthrosis & 13.0 & 10.7 & 0.0510 \\
\hline Cerebrovascular disease & 11.6 & 11.7 & 0.9721 \\
\hline Depression & 9.9 & 8.9 & 0.3838 \\
\hline Vasculitis & 9.0 & 8.8 & 0.8217 \\
\hline Osteoporosis & 8.8 & 6.7 & 0.0297 \\
\hline Pneumonia & 8.0 & 5.5 & 0.0042 \\
\hline Hypothyroidism & 7.9 & 6.7 & 0.2013 \\
\hline Hypercholesterolemia & 7.7 & 8.0 & 0.7434 \\
\hline Diverticulosis & 7.6 & 9.2 & 0.1187 \\
\hline Gastroesophageal reflux disease & 6.5 & 4.6 & 0.0144 \\
\hline Chronic hepatitis & 6.0 & 5.5 & 0.7241 \\
\hline Respiratory failure & 5.4 & 3.0 & 0.0005 \\
\hline Cholelithiasis & 5.4 & 3.6 & 0.0159 \\
\hline Gallstones & 5.3 & 4.2 & 0.2756 \\
\hline Carotid Atherosclerosis & 4.9 & 5.6 & 0.6553 \\
\hline Anxiety & 4.9 & 5.3 & 0.6430 \\
\hline Hepatic cirrhosis & 3.5 & 4.9 & 0.0694 \\
\hline
\end{tabular}

\begin{tabular}{llll}
\hline Variables & Inpatient with COPD & Inpatient without COPD & $p$ \\
\hline Length of hospital stay (days) $_{\text {In hospital mortality (\%) }}$ & $11.82(11.21-12.43)$ & $11.80(11.34-12.25)$ & 0.0183 \\
3-month mortality (\%) & 5.5 & 5.4 & 0.9293 \\
12-month mortality (\%) & 9.6 & 9.4 & 0.8573 \\
Destination at discharge (3-month) & 13.5 & 14.1 & 0.8392 \\
$\quad$ Home (\%) & 88.5 & & \\
Home care (\%) & 3.4 & 89.4 & 0.5386 \\
Institution (\%) & 3.4 & 3.2 & 0.7963 \\
Rehospitalization (\%) & 4.7 & 4.2 & 0.4098 \\
Destination at discharge (12-month) & & 3.2 & 0.0959 \\
$\quad$ Home (\%) & 86.5 & 89.6 & 0.3383 \\
Home care (\%) & 3.4 & 2.5 & 0.6200 \\
Institution (\%) & 5.0 & 5.7 & 0.7668 \\
Rehospitalization (\%) & 21.0 & 13.3 & 0.0261 \\
\hline
\end{tabular}

${ }^{a}$ Data are reported as means (95\% confidence interval) 
gastroesophageal reflux disease, respiratory failure, and cholelithiasis were more frequent in COPD patients (Table 3).

As shown in Table 4, subjects with COPD had significantly longer hospital stay; in addition, higher rates of rehospitalization at 1-year after discharge were recorded.

In-hospital and within 1-year mortality did not differ between the two groups. However, when we assessed independent predictors of mortality, running univariate analysis (see appendix) and then multivariate analysis (Fig. 1) according to Hosmer-Lemeshow methodology, cerebrovascular disease and current ICS-LABA therapy were independently associated with in-hospital mortality. At 3-month follow-up, male gender, hepatic cirrhosis, and ICS-LABA therapy were predictors of mortality. At 1-year follow-up, ICS-LABA therapy was the only predictor of mortality. COPD exacerbation did not represent an independent predictor of mortality in older hospitalized people even if $43 \%$ of patients with COPD had exacerbation.

\section{Discussion}

In this observational study on the RePoSI registry, we assessed the distribution of comorbidities and the occurrence of outcomes in a population of elderly COPD in-patients admitted to the internal medicine and geriatric wards, with the aim to evaluate whether COPD subjects behave differently from non-COPD individuals. Overall, the current findings suggest that COPD subjects are at higher risk of death within the first year from admission to the hospital.

Although comorbidities are increasingly identified as important factors of COPD management and outcomes [15], studies specifically designed to evaluate the relationships between comorbidities and long-term outcomes in subjects with a diagnosis of COPD admitted to an internal medicine ward are scarce [16], and this is also true for several chronic diseases [17]. A recent study showed that the addition of comorbidities to age, BMI, blood markers and indexes such as smoking status, dyspnea assessment, airway obstruction

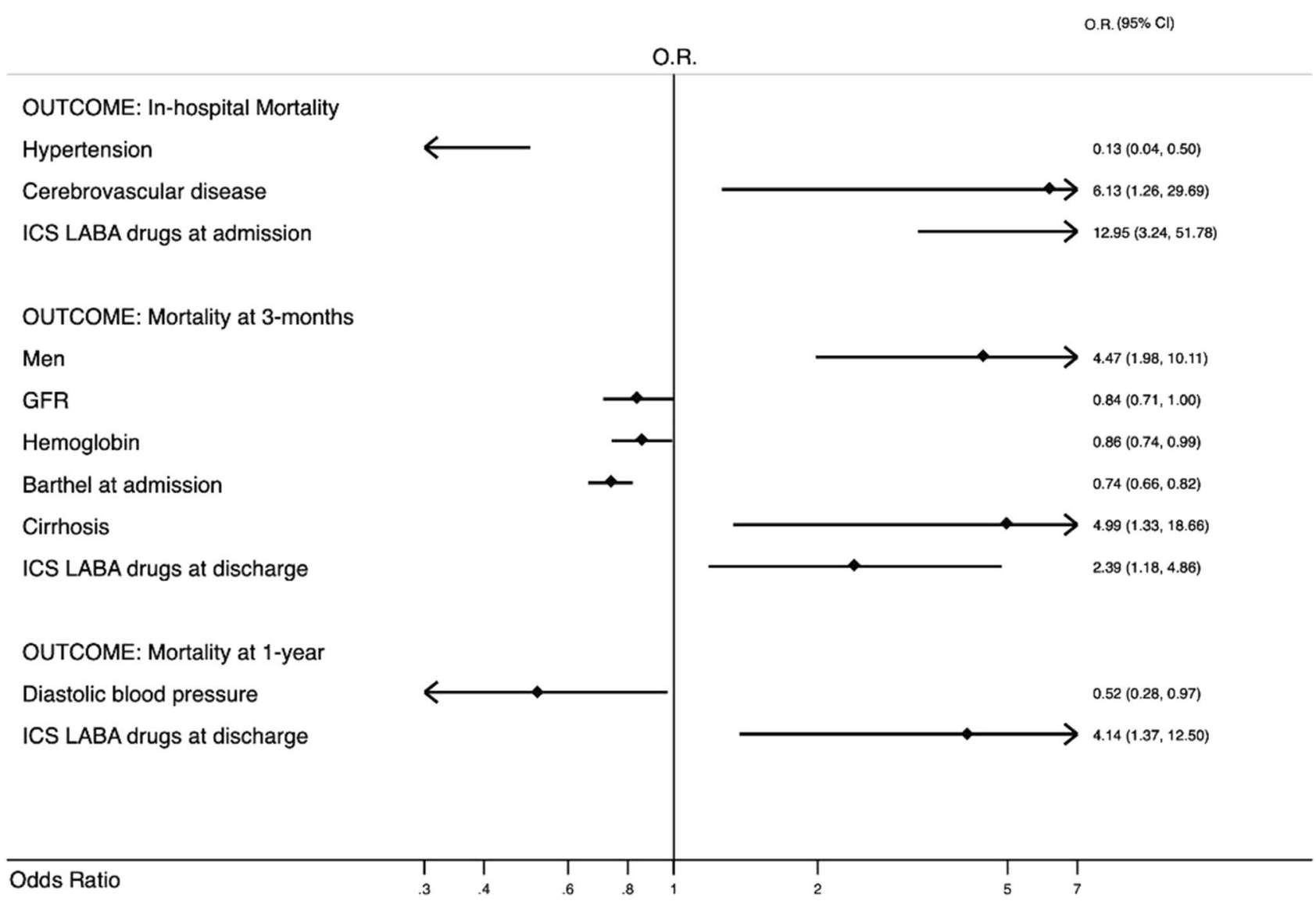

Fig. 1 Multivariate Analysis in COPD patients according to in-hospital, 3-month and 1-year mortality. OR odds ratio, 95\% CI 95\% confidence interval, ICS/LABA Inhaled corticosteroids and long-acting beta2-agonists in combination, GFR Glomerular Filtration Rate calculated by CKD-EPI formula; GFR is referred to values every $10 \mathrm{ml} /$ min; Barthel Index is referred to values every 10 points; Diastolic Blood Pressure is referred to values every $10 \mathrm{mmHg}$. Only the final model is shown according to Hosmer-Lemeshow methodology. For the selection of variables see appendix and statistical analysis section 
produced a model, known as BARC index, that performed better than established index scores in predicting 1-year mortality [18]. Our analysis showed that COPD in-patients are more often older men, smokers or former smokers, and live with their relatives, in agreement with our previous findings [19]. Moreover, COPD patients are severe obese consistently with recent data that seem to confirm that obesity is more common in COPD patients compared to subjects who do not have COPD [20]. Interestingly, individuals with a diagnosis of COPD had more frequent mood changes, indicating higher level of distress, in agreement with those from the NHANES study of $20.6 \%$ of subjects with COPD suffering from depression [21]. Shane et al. showed that up to $40 \%$ of patients with COPD had clinically significant depressive symptoms, a proportion higher than that recorded in other chronic diseases such as stroke, diabetes, coronary heart disease, arthritis, hypertension, and cancer [22]. Similarly, COPD patients showed worse cognitive impairment than non-COPD patients; in the study by Dodd et al., up to $57 \%$ of patients with COPD exacerbation had features of cognitive impairment [23]. A recent systematic review and meta-analyses outlined that one in four subjects with COPD has mild cognitive impairment [24]. In addition to affecting pharmacological treatment, comorbidities may impair the ability to use inhalation devices [25]; for example, cognitive impairments affect the ability to properly use the inhaled device, and anxiety and depression can reduce the adherence to treatment. It follows that the choice of the proper inhaler should also take into account the relative contribution of concomitant diseases in affecting the correct use of the device. It is commonly accepted that cognitive impairment and depression lead to progressive disability $[19,26]$, especially in oldest-old subjects [27, 28], thus potentially affecting short-and long-term outcomes. The current findings also show that the presence of anemia is associated with the frequency of exacerbations and increasing healthcare costs [29, 30]. The phenomenon is relevant in clinical practice: indeed, Cote et al. found that anemia was present in 17\% of COPD inpatients [31]. The possible mechanism consists in persistent elevated interleukin levels, in particular IL-1, that interfere with the erythropoietin response [32].

The current analysis highlighted that COPD patients had a worse functional status than patients without COPD; this is of clinical importance, given that hospitalized elderly patients affected by pneumonia with a clinically significant disability were already shown to have higher mortality risk [33]. Lanièce et al. found that severe disability was the most important predictor of early re-admission among elderly inpatients [34]. Recent data showed that male gender, previously hospitalized, polypharmacy (more than 5 drugs), lower functional status and frailty, depression, heart diseases, COPD, urinary tract infection were associated to a higher risk of hospitalization
[35]. Moreover, heart failure, diabetes and stroke were associated with a prolonged hospital stay ( $>11$ days) in hospitalized COPD patients [36]. The current findings on comorbidities distribution showed a significant prevalence of respiratory failure and respiratory conditions other than COPD, as well as cardiovascular diseases, chronic renal failure, prostatic hypertrophy, rheumatic diseases, and gastroesophageal reflux disease. An interesting speculation on these findings comes from the theory of network medicine [37], based on which human diseases are not independent of each other, but rather the consequence of different biological processes that interact in this complex network, defined as "diseasome". In this regard, COPD is among the best scenario in which multiple factors such as chronic inflammation, aging-related changes, altered immune response, increased oxidative stress, consequences of smoke exposure and physical inactivity are variably interwound. Aging per se is characterized by chronic low-grade systemic inflammation, and is associated with multiple chronic conditions, including COPD [2, 38, 39]; interestingly, a relationship among systemic inflammation, comorbidities and COPD outcomes has been clearly documented [40]. Of note, ischemic heart disease, heart failure, myocardial infarction, diabetes, lung cancer, osteoporosis, metabolic syndrome, are all characterized by low-grade inflammation and frequently associated with COPD [41].

The question is whether, and to what extent, comorbidities affect mortality independent of lung disease. Using data from the multicenter observational study ECLIPSE, Agusti and colleagues [42] proposed the systemic inflammome, a network representation of systemic inflammation in individuals suffering from COPD, which may account, in a proportion of subjects who are persistently inflamed, for significantly higher rates of all-cause mortality. The prevalence of comorbidities in patients with COPD was assessed by Divo and collaborators [5], who identified specific comorbidities significantly associated with increased mortality. The relative contribution of each comorbidity to mortality and the relationships among comorbidities led to the so-called "comorbidome". Vanfleteren et al. [43] identified five clusters of comorbidities: "cardiovascular", "cachectic", "metabolic" and "psychological" and "less comorbidity". The authors however failed to find any association with mortality. Our findings indicate that cerebrovascular disease significantly increased the risk of death during hospitalization. On the other hand, cirrhosis and men gender were significantly associated with 3-month mortality. These observations are in agreement with Kim et al. that found a significant statistically association between COPD and increased risk of stroke [44], and with Divo et al. that found that the risk of death was strongly associated with different comorbidities including liver cirrhosis, suggesting a correlation with lifestyle and social behavior [5]. These data were also confirmed by Baty 
et al. that found a higher prevalence of alcoholic cirrhosis in their nationwide analysis of hospital admissions for COPD in Switzerland [45]. Moreover, our results are consistent with previous studies that identified comorbidities that were associated with COPD progression and exacerbation frequency, poor quality of life, higher mortality and increase of costs management $[5,46,47]$. The current analysis highlights the role of the ICS-LABA regular treatment, which was independently associated with in-hospital, 3-month and 1-year follow-up mortality. This result was unchanged even if variables such as COPD exacerbation, heart failure, atrial fibrillation, ischemic heart disease, oral anticoagulants, antiplatelet drugs had been included into the model. In a recent meta-analysis, Horita et al. found that patients treated with LABA-LAMA had fewer exacerbations and a significantly lower risk of developing pneumonia in comparison with ICS-LABA [48].

In addition, Ernst et al. suggested a limit use of ICS and ICS-LABA in COPD patients on the basis of the evidence of adverse effects, especially severe pneumonia, leading to excess mortality [49]. Although the causes of mortality are not known, it cannot be excluded that chronic use of ICS was responsible for severe adverse events in compromised subjects. The lack of data on the dosage or the class of corticosteroids does not allow to draw firm conclusions on the contribution of the active drug. Similarly, it is plausible to hypothesize that LABA variably influenced the outcome.

A recent study showed the importance of $\mathrm{BI}$ as a strong predictor of 30-days, 3-and 12-month mortality in elderly patients with pneumonia [33]. Simonetti et al. found that pneumonia severity and low functional status are the main factors associated with mortality in elderly people with community acquired pneumonia [50]. Vitacca et al. suggested the utilization of a unique instrument, i.e. the BI-dyspnea, to provide a global assessment of disability evaluating both respiratory and motor impairment [51]. Formiga et al. demonstrated that a better functional status and a lower comorbidity conditions were independent predictors of mortality at 5-years in 85-year-old community-dwelling subjects [52]. In the current study, disability did not enter the multivariate analysis as independent predictor of mortality, although the Barthel score suggestive of physical impairment clearly distinguished the COPD phenotype (Fig. 1).

A possible explanation for the apparent discrepancy between studies lies in the lack of information on the lung functional impairment, which may variably affect the ability to interact with daily activities. It is therefore logical to hypothesize that disability is one of the strongest predictors of mortality also in COPD. Further studies are needed to confirm it.

With regard to the protective function of higher glomerular filtration rate, our data are consistent with those of Singanayagam et al. who established that chronic renal failure was significantly associated with increased short-term mortality in patients with COPD [53]. A potential explanation lies in the glomerular damage by arterial stiffness along with hypoxic damage to tubules and interstitium as possible mechanisms in the relationship between COPD and chronic renal failure [54].

We found that blood pressure had a protective role regarding in-hospital mortality. Our findings are in agreement with previous observations that showed a reverse association between higher blood pressure and mortality in oldest old patients [55, 56]. Moreover, recent analysis showed that in contrast to the general population, in frail elderly patients increased blood pressure is associated with reduced mortality. A possible explanation is that high blood pressure is necessary to maintain sufficient organ perfusion in a population of older subjects who are likely to have significant vascular damage [57, 58].

Regarding sex, our results are consistent with a previous study that showed in elderly hospitalized patients a male profile, smokers or former smokers, affected by COPD, coronary artery disease and cancer responsible for re-hospitalization and higher mortality [19, 59].

This observational study has some limits. First, there was no specific information about how the diagnosis of COPD was formulated (GOLD criteria, radiological criteria), and the severity of COPD was not taken into account. Given the lack of spirometric confirmation, it cannot be excluded that a proportion of subjects actually suffered from chronic diseases other than COPD. However, the observational nature of the design and the exploratory approach limit the weaknesses of the findings. Second, the lack of information on the appropriateness of prescriptions, and the opportunity to exclude potential confounders that goes beyond the scope of the RePoSI study. The major strength of the study is the multicenter design of the RePoSI register and the large number of participating centers resulting in a comprehensive sample of the elderly patients hospitalized in internal medicine and geriatric wards.

In conclusion, this study showed the impact of COPD and its comorbidities in the real-world scenario of internal and geriatric wards, identifying factors that are linked with short-and long-term outcomes. The current findings strongly support that the management of COPD patients should include identification and treatment of its comorbidities. This approach should be the first step for personalized care based on a multidimensional assessment of elderly patients affected by COPD.

Electronic supplementary material The online version of this article (https://doi.org/10.1007/s11739-020-02412-1) contains supplementary material, which is available to authorized users.

Acknowledgements Investigators and co-authors of the REPOSI ( istro POliterapie SIMI, Società Italiana di Medicina Interna) Study 
Group are as follows: Steering Committee: Pier Mannuccio Mannucci (Chair) (Fondazione IRCCS Cà Granda Ospedale Maggiore Policlinico, Milano), Alessandro Nobili (co-chair) (Istituto di Ricerche Farmacologiche Mario Negri IRCCS, Milano), Antonello Pietrangelo (Presidente SIMI), Francesco Perticone (Direttore CRIS - SIMI), Giuseppe Licata (Socio d'onore SIMI), Francesco Violi (Policlinico Umberto I, Roma, Prima Clinica Medica), Gino Roberto Corazza, (Reparto 11, IRCCS Policlinico San Matteo di Pavia, Pavia, Clinica Medica I), Salvatore Corrao (ARNAS Civico, Di Cristina, Benfratelli, DiBiMIS, Università di Palermo, Palermo), Alessandra Marengoni (Spedali Civili di Brescia, Brescia), Francesco Salerno (IRCCS Policlinico San Donato Milanese, Milano), Matteo Cesari (UO Geriatria, Università degli Studi di Milano), Mauro Tettamanti, Luca Pasina, Carlotta Franchi (Istituto di Ricerche Farmacologiche Mario Negri IRCCS, Milano). Clinical data monitoring and revision: Carlotta Franchi, Laura Cortesi, Mauro Tettamanti, Gabriella Miglio (Istituto di Ricerche Farmacologiche Mario Negri IRCCS, Milano). Database Management and Statistics: Mauro Tettamanti, Laura Cortesi, Ilaria Ardoino, Alessio Novella (Istituto di Ricerche Farmacologiche Mario Negri IRCCS, Milano). Investigators: Domenico Prisco, Elena Silvestri, Giacomo Emmi, Alessandra Bettiol, Cenci Caterina (Azienda Ospedaliero Universitaria Careggi Firenze, Medicina Interna Interdisciplinare); Gianni Biolo, Michela Zanetti, Martina Guadagni, Michele Zaccari, Massimiliano Chiuch, Michele Zaccari (Azienda Sanitaria Universitaria Integrata di Trieste, Clinica Medica Generale e Terapia Medica); Massimo Vanoli, Giulia Grignani, Edoardo Alessandro Pulixi (Azienda Ospedaliera della Provincia di Lecco, Ospedale di Merate, Lecco, Medicina Interna); Mauro Bernardi, Silvia Li Bassi, Luca Santi, Giacomo Zaccherini (Azienda Ospedaliera Policlinico Sant'Orsola-Malpighi, Bologna, Semeiotica Medica Bernardi); Graziana Lupattelli, Elmo Mannarino, Vanessa Bianconi, Francesco Paciullo, Riccardo Alcidi (Azienda Ospedaliera Santa Maria della Misericordia, Perugia, Medicina Interna); Ranuccio Nuti, Roberto Valenti, Martina Ruvio, Silvia Cappelli, Alberto Palazzuoli (Azienda Ospedaliera Università Senese, Siena, Medicina Interna I); Domenico Girelli, Fabiana Busti, Giacomo Marchi (Azienda Ospedaliera Universitaria Integrata di Verona, Verona, Medicina Generale e Malattie Aterotrombotiche e Degenerative); Mario Barbagallo, Ligia Dominguez, Floriana Cocita, Vincenza Beneduce, Lidia Plances (Azienda Ospedaliera Universitaria Policlinico Giaccone Policlinico di Palermo, Palermo, Unità Operativa di Geriatria e Lungodegenza); Salvatore Corrao, Giuseppe Natoli, Salvatore Mularo, Massimo Raspanti, Federica Cavallaro (A.R.N.A.S. Civico, Di Cristina, Benfratelli, Palermo, UOC Medicina Interna ad Indirizzo Geriatrico-Riabilitativo); Marco Zoli, Ilaria Lazzari, Mattia Brunori, Elisa Fabbri, Donatella Magalotti, Raffaella Arnò (Azienda Ospedaliera Universitaria Policlinico S. Orsola-Malpighi, Bologna, Unità Operativa di Medicina Interna); Franco Laghi Pasini, Pier Leopoldo Capecchi, (Azienda Ospedaliera Universitaria Senese, Siena, Unità Operativa Complessa Medicina 2); Giuseppe Palasciano, Maria Ester Modeo, Carla Di Gennaro (Azienda Ospedaliero-Universitaria Consorziale Policlinico di Bari, Bari, Medicina Interna Ospedaliera “L. D’Agostino”, Medicina Interna Universitaria “A. Murri”); Maria Domenica Cappellini, Diletta Maira, Valeria Di Stefano, Giovanna Fabio, Sonia Seghezzi, Marta Mancarella, Margherita Migone De Amicis, Giacomo De Luca, Natalia Scaramellini (Fondazione IRCCS Cà Granda Ospedale Maggiore Policlinico, Milano, Unità Operativa Medicina Interna IA); Matteo Cesari, Paolo Dionigi Rossi, Sarah Damanti, Marta Clerici, Federica Conti, Giulia Bonini, Barbara Brignolo Ottolini (Fondazione IRCCS Cà Granda Ospedale Maggiore Policlinico, Milano, Geriatria); Antonio Di Sabatino, Emanuela Miceli, Marco Vincenzo Lenti, Martina Pisati, Costanza Caccia Dominioni (IRCCS Policlinico San Matteo di Pavia, Pavia, Clinica Medica I, Reparto 11); Giovanni Murialdo, Alessio Marra, Federico Cattaneo, Roberto Pontremoli, Valentina Beccati, Giulia Nobili (IRCCS Azienda Ospedaliera Universitaria San MartinoIST di Genova, Genova, Clinica di Medicina Interna 2); Maria Beatrice Secchi, Davide Ghelfi (Ospedale Bassini di Cinisello Balsamo, Milano,
Divisione Medicina); Luigi Anastasio, Lucia Sofia, Maria Carbone (Ospedale Civile Jazzolino di Vibo Valentia, Vibo Valentia, Medicina interna); Francesco Cipollone, Maria Teresa Guagnano, Emanuele Valeriani, Ilaria Rossi (Ospedale Clinicizzato SS. Annunziata, Chieti, Clinica Medica); Gerardo Mancuso, Daniela Calipari, Mosè Bartone (Ospedale Giovanni Paolo II Lamezia Terme, Catanzaro, Unità Operativa Complessa Medicina Interna); Giuseppe Delitala, Maria Berria, Chiara Pes, Alessandro Delitala (Azienda ospedaliera-universitaria di Sassari, Clinica Medica); Maurizio Muscaritoli, Alessio Molfino, Enrico Petrillo (Policlinico Umberto I, Sapienza Università di Roma, Medicina Interna e Nutrizione Clinica Policlinico Umberto I); Giuseppe Zuccalà, Gabriella D'Aurizio (Policlinico Universitario A. Gemelli, Roma, Roma, Unità Operativa Complessa Medicina d'Urgenza e Pronto Soccorso); Giuseppe Romanelli, Alessandra Marengoni, Alberto Zucchelli, Francesca Manzoni, Andrea Volpini (Spedali Civili di Brescia, Brescia, Geriatria); Antonio Picardi, Umberto Vespasiani Gentilucci, Paolo Gallo, Chiara Dell'Unto (Università Campus Bio-Medico, Roma, Medicina Clinica-Epatologia); Giorgio Annoni, Maurizio Corsi, Giuseppe Bellelli, Sara Zazzetta, Paolo Mazzola, Hajnalka Szabo, Alessandra Bonfanti (Università degli studi di Milano-Bicocca Ospedale S. Gerardo, Monza, Unità Operativa di Geriatria); Franco Arturi, Elena Succurro, Mariangela Rubino, Bruno Tassone, Giorgio Sesti (Università degli Studi Magna Grecia, Policlinico Mater Domini, Catanzaro, Unità Operativa Complessa di Medicina Interna); Maria Grazia Serra, Maria Antonietta Bleve (Azienda Ospedaliera "Cardinale Panico" Tricase, Lecce, Unità Operativa Complessa Medicina); Laura Gasbarrone, Maria Rosaria Sajeva (Azienda Ospedaliera Ospedale San Camillo Forlanini, Roma, Medicina Interna 1); Antonio Brucato, Silvia Ghidoni (Azienda Ospedaliera Papa Giovanni XXIII, Bergamo, Medicina 1); Fabrizio Fabris, Irene Bertozzi, Giulia Bogoni, Maria Victoria Rabuini, Elisabetta Cosi, Paolo Scarinzi, Annalisa Amabile, Elisabetta Omenetto, Tancredi Prandini (Azienda Ospedaliera Università di Padova, Padova, Clinica Medica I); Roberto Manfredini, Fabio Fabbian, Benedetta Boari, Alfredo De Giorgi, Ruana Tiseo, Roberto De Giorgio (Azienda Ospedaliera Universitaria Sant'Anna, Ferrara, Unità Operativa Clinica Medica); Giuseppe Paolisso, Maria Rosaria Rizzo (Azienda Ospedaliera Universitaria della Seconda Università degli Studi di Napoli, Napoli, VI Divisione di Medicina Interna e Malattie Nutrizionali dell'Invecchiamento); Claudio Borghi, Enrico Strocchi, Eugenia Ianniello, Mario Soldati (Azienda Ospedaliera Universitaria Policlinico S. Orsola-Malpighi, Bologna, Unità Operativa di Medicina Interna Borghi); Carlo Sabbà, Francesco Saverio Vella, Patrizia Suppressa, Andrea Schilardi, Francesca Loparco, Giovanni Michele De Vincenzo, Alessio Comitangelo, Emanuele Amoruso (Azienda Ospedaliero-Universitaria Consorziale Policlinico di Bari, Bari, Medicina Interna Universitaria C. Frugoni); Luigi Fenoglio, Andrea Falcetta, Christian Bracco (Azienda Sanitaria Ospedaliera Santa Croce e Carle di Cuneo, Cuneo, S. C. Medicina Interna); Anna L. Fracanzani Silvia Fargion,, Silvia Tiraboschi, Annalisa Cespiati, Giovanna Oberti, Giordano Sigon (Fondazione IRCCS Cà Granda Ospedale Maggiore Policlinico, Milano, Medicina Interna 1B); Flora Peyvandi, Raffaella Rossio, Barbara Ferrari, Giulia Colombo, Pasquale Agosti (Fondazione IRCCS Cà Granda Ospedale Maggiore Policlinico, Milano, UOC Medicina generale - Emostasi e trombosi); Valter Monzani, Valeria Savojardo, Christian Folli, Giuliana Ceriani (Fondazione IRCCS Cà Granda Ospedale Maggiore Policlinico, Milano, Medicina Interna Alta Intensità); Francesco Salerno, Giada Pallini (IRCCS Policlinico San Donato e Università di Milano, San Donato Milanese, Medicina Interna); Franco Dallegri, Luciano Ottonello, Luca Liberale, Lara Caserza, Kassem Salam (Università di Genova, Genova, Medicina Interna 1); Nicola Lucio Liberato, Tiziana Tognin (ASST di Pavia, UOSD Medicina Interna, Ospedale di Casorate Primo, Pavia); Giovanni Battista Bianchi, Sabrina Giaquinto (Ospedale "SS Gerosa e Capitanio" di Lovere, Bergamo, Unità Operativa Complessa di Medicina Generale, Azienda Ospedaliera "Bolognini" di Seriate, Bergamo); Francesco Purrello, Antonino Di Pino, Salvatore Piro 
(Ospedale Garibaldi Nesima, Catania, Unità Operativa Complessa di Medicina Interna); Renzo Rozzini, Lina Falanga, Elena Spazzini, Camillo Ferrandina (Ospedale Poliambulanza, Brescia, Medicina Interna e Geriatria); Giuseppe Montrucchio, Paolo Petitti, Paolo Peasso, Edoardo Favale, Cesare Poletto (Dipartimento di Scienze Mediche, Università di Torino, Città della Scienza e della Salute, Torino, Medicina Interna 2 U. Indirizzo d'Urgenza); Raffaella Salmi, Piergiorgio Gaudenzi (Azienda Ospedaliera-Universitaria S. Anna, Ferrara, Unità Operativa di Medicina Ospedaliera II); Francesco Violi, Ludovica Perri (Policlinico Umberto I, Roma, Prima Clinica Medica); Raffaele Landolfi, Massimo Montalto, Antonio Mirijello (Policlinico Universitario A. Gemelli, Roma, Clinica Medica); Luigina Guasti, Luana Castiglioni, Andrea Maresca, Alessandro Squizzato, Leonardo Campiotti, Alessandra Grossi (Università degli Studi dell'Insubria, Ospedale di Circolo e Fondazione Macchi, Varese, Medicina Interna I); Marco Bertolotti, Chiara Mussi, Giulia Lancellotti, Maria Vittoria Libbra, Giulia Dondi, Elisa Pellegrini, Lucia Carulli, Matteo Galassi, Yasmine Grassi (Università diModena e Reggio Emilia, Azienda Ospedaliero-Universitaria di Modena; Ospedale Civile di Baggiovara, Unità Operativa di Geriatria); Francesco Perticone, Maria Perticone, Rosa Battaglia, Marco Fllice, Raffaele Maio (Università Magna Grecia Policlinico Mater Domini, Catanzaro, Unità Operativa Malattie Cardiovascolari Geriatriche); Vincenzo Stanghellini, Eugenio Ruggeri, Sara del Vecchio (Dipartimento di Scienze Mediche e Chirurgiche, Unità Operativa di Medicina Interna, Università degli Studi di Bologna/Azienda Ospedaliero-Universitaria S.Orsola-Malpighi, Bologna); Andrea Salvi, Roberto Leonardi, Giampaolo Damiani (Spedali Civili di Brescia, U.O. 3a Medicina Generale); William Capeci, Armando Gabrielli, Massimo Mattioli, Giuseppe Pio Martino, Lorenzo Biondi, Pietro Pettinari (Clinica Medica, Azienda Ospedaliera Universitaria - Ospedali Riuniti di Ancona); Riccardo Ghio, Anna Dal Col (Azienda Ospedaliera Università San Martino, Genova, Medicina III); Salvatore Minisola, Luciano Colangelo, Mirella Cilli, Giancarlo Labbadia (Policlinico Umberto I, Roma, SMSC03 - Medicina Interna A e Malattie Metaboliche dell'osso); Antonella Afeltra, Benedetta Marigliano, Maria Elena Pipita (Policlinico Campus Biomedico Roma, Roma, Medicina Clinica); Pietro Castellino, Luca Zanoli, Samuele Pignataro, Alfio Gennaro, Julien Blanco (Azienda Ospedaliera Universitaria Policlinico - V. Emanuele, Catania, Dipartimento di Medicina); Valter Saracco, Marisa Fogliati, Carlo Bussolino (Ospedale Cardinal Massaia Asti, Medicina A); Francesca Mete, Miriam Gino (Ospedale degli Infermi di Rivoli, Torino, Medicina Interna) Antonio Cittadini, Carlo Vigorito, Michele Arcopinto, Andrea Salzano, Emanuele Bobbio, Alberto Maria Marra, Domenico Sirico (Azienda Policlinico Universitario Federico II di Napoli, Napoli, Medicina Interna e Riabilitazione Cardiologica); Guido Moreo, Francesca Gasparini, Silvia Prolo, Gloria Pina (Clinica San Carlo Casa di Cura Polispecialistica, Paderno Dugnano, Milano, Unità Operativa di Medicina Interna); Alberto Ballestrero, Fabio Ferrando (Clinica Di Medicina Interna ad Indirizzo Oncologico, Azienda Ospedaliera Università San Martino di Genova); Sergio Berra, Simonetta Dassi, Maria Cristina Nava (Medicina Interna, Azienda Ospedaliera Guido Salvini, Garnagnate, Milano); Bruno Graziella, Stefano Baldassarre, Salvatore Fragapani, Gabriella Gruden (Medicina Interna III, Ospedale S. Giovanni Battista Molinette, Torino); Giorgio Galanti, Gabriele Mascherini, Cristian Petri, Laura Stefani (Agenzia di Medicina dello Sport, AOUC Careggi, Firenze); Margherita Girino, Valeria Piccinelli (Medicina Interna, Ospedale S. Spirito Casale Monferrato, Alessandria); Francesco Nasso, Vincenza Gioffrè, Maria Pasquale (Struttura Operativa Complessa di Medicina Interna, Ospedale Santa Maria degli Ungheresi, Reggio Calabria); Giuseppe Scattolin, Sergio Martinelli, Mauro Turrin (Medicina Interna, Ospedale di Monselice, Padova); Leonardo Sechi, Cristina Catena, Gianluca Colussi (Clinica Medica, Azienda Ospedaliera Universitaria, Udine). Nicola Passariello, Luca Rinaldi (Presidio Medico di Marcianise, Napoli, Medicina Interna); Franco Berti, Giuseppe Famularo, Patrizia Tarsitani (Azienda Ospedaliera San Camillo Forlanini, Roma, Medicina Interna
II); Roberto Castello, Michela Pasino (Ospedale Civile Maggiore Borgo Trento, Verona, Medicina Generale e Sezione di Decisione Clinica); Gian Paolo Ceda, Marcello Giuseppe Maggio, Simonetta Morganti, Andrea Artoni (Azienda Ospedaliero Universitaria di Parma, U.O.C Clinica Geriatrica); Stefano Del Giacco, Davide Firinu, Francesca Losa, Giovanni Paoletti, Giulia Costanzo (Policlinico Universitario Duilio Casula, Azienda Ospedaliero-Universitaria di Cagliari, Cagliari, Medicina Interna, Allergologia ed Immunologia Clinica); Giuseppe Montalto, Anna Licata, Valentina Malerba, Filippo Alessandro Montalto (Azienda Ospedaliera Universitaria Policlinico Paolo Giaccone, Palermo, UOC di Medicina Interna); Antonino Lasco, Giorgio Basile, Antonino Catalano (Azienda Ospedaliera Universitaria Policlinico G. Martino, Messina, Unità Operativa di Geriatria); Lorenzo Malatino, Benedetta Stancanelli, Valentina Terranova, Salvatore Di Marca, Rosario Di Quattro, Lara La Malfa, Rossella Caruso (Azienda Ospedaliera per l'Emergenza Cannizzaro, Catania, Clinica Medica Università di Catania); Patrizia Mecocci, Carmelinda Ruggiero, Virginia Boccardi (Università degli Studi di Perugia-Azienda Ospedaliera S.M. della Misericordia, Perugia, Struttura Complessa di Geriatria); Tiziana Meschi, Fulvio Lauretani, Andrea Ticinesi, Antonio Nouvenne (Azienda Ospedaliera Universitaria di Parma, U.O Medicina Interna e Lungodegenza Critica); Pietro Minuz, Luigi Fondrieschi (Azienda Ospedaliera Universitaria Verona, Policlinico GB Rossi, Verona, Medicina Generale per lo Studio ed il Trattamento dell'Ipertensione Arteriosa); Mario Pirisi, Gian Paolo Fra, Daniele Sola (Azienda Ospedaliera Universitaria Maggiore della Carità, Medicina Interna 1); Massimo Porta, Piero Riva (Azienda Ospedaliera Universitaria Città della Salute e della Scienza di Torino, Medicina Interna 1U); Roberto Quadri, Erica Larovere, Marco Novelli (Ospedale di Ciriè, ASL TO4, Torino, S.C. Medicina Interna); Giorgio Scanzi, Caterina Mengoli, Stella Provini, Laura Ricevuti (ASST Lodi, Presidio di Codogno, Milano, Medicina); Emilio Simeone, Rosa Scurti, Fabio Tolloso (Ospedale Spirito Santo di Pescara, Geriatria); Roberto Tarquini, Alice Valoriani, Silvia Dolenti, Giulia Vannini (Ospedale San Giuseppe, Empoli, USL Toscana Centro, Firenze, Medicina Interna I); Alberto Tedeschi, Lucia Trotta (ASST Fatebenefratelli - Sacco, Milano, Medicina Interna a indirizzo Pneumologico); Riccardo Volpi, Pietro Bocchi, Alessandro Vignali (Azienda Ospedaliera Universitaria di Parma, Clinica e Terapia Medica). Sergio Harari, Chiara Lonati, Mara Cattaneo, Federico Napoli (Ospedale San Giuseppe Multimedica Spa, U.O. Medicina Generale).

\section{Compliance with ethical standards}

Conflict of interest The authors report no conflicts of interest.

Statement of human and animal rights The study including human participants has been performed in accordance with the ethical standards of the Declaration of Helsinki and its later amendments.

Informed consent Informed consent was obtained from all patients prior to all surgical procedures.

\section{References}

1. Mathers CD, Loncar D (2006) Projections of global mortality and burden of disease from 2002 to 2030. PLoS Med. 3:e442

2. Fabbri LM, Luppi F, Beghe B et al (2008) Complex chronic comorbidities of COPD. Eur Respir J 31:204-212

3. Agusti A, Calverley PM, Celli B et al (2010) Characterisation of COPD heterogeneity in the ECLIPSE cohort. Respir Res 11:122 
4. Cavaillès A, Brinchault-Rabin G, Dixmier A et al (2013) Comorbidities of COPD. Eur Respir Rev 22:454-475

5. Divo M, Cote C, de Torres JP, BODE Collaborative Group et al (2012) Comorbidities and risk of mortality in patients with chronic obstructive pulmonary disease. Am J Respir Crit Care Med 186(2):155-161

6. Battaglia S, Basile M, Spatafora M et al (2015) Are asthmatics enrolled in randomized trials representative of real-life outpatients? Respiration 89:383-389

7. Scichilone N, Basile M, Battaglia S et al (2014) What proportion of chronic obstructive pulmonary disease outpatients is eligible for inclusion in randomized clinical trials? Respiration 87(1):11-17

8. The REPOSI registry of multimorbidity and polypharmacy: http:// www.reposi.org. Accessed 12 Dec 2018

9. Katzman R, Brown T, Fuld P et al (1983) Validation of a short Orientation-Memory-Concentration Test of cognitive impairment. Am J Psychiatry 140:734-739

10. Hickie C, Snowdon J (1987) Depression scales for the elderly: gDS, Gilleard, Zung. Clin Gerontol J Aging Ment Health. 6:51-53

11. Mahoney FI, Barthel DW (1965) Functional evaluation: the Barthel index. Md State Med J. 14:61-65

12. Linn BS, Linn MW, Gurel L (1968) Cumulative illness rating scale. J Am Geriatr Soc 16:622-626

13. Levey AS, Stevens LA, Schmid CH, CKD-EPI (Chronic Kidney Disease Epidemiology Collaboration) (2009) A new equation to estimate glomerular filtration rate. Ann Intern Med. 150:604-612

14. Hosmer DW, Lemeshow S (2000) Multiple logistic regression. In: Hosmer DW, Lemeshow S (eds) Applied logistic regression, 2nd edn. J Wiley; New York, NY, pp 31-46

15. Aramburu A, Arostegui I, Moraza J et al (2019) COPD classification models and mortality prediction capacity. Int J Chron Obstruct Pulmon Dis. 14:605-613

16. Yin HL, Yin SQ, Lin QY et al (2017) Prevalence of comorbidities in chronic obstructive pulmonary disease patients: a metaanalysis. Medicine 96(19):e6836

17. Almagro P, Cabrera FJ, Diez J, Working Group on, COPD, Spanish Society of Internal Medicine et al (2012) Comorbidities and short-term prognosis in patients hospitalized for acute exacerbation of COPD: the EPOC en Servicios de medicina interna (ESMI) study. Chest 142(5):1126-1133

18. Bloom CI, Ricciardi F, Smeeth L, Stone P, Quint JK (2019) Predicting COPD 1-year mortality using prognostic predictors routinely measured in primary care. BMC Med. 17(1):73

19. Corrao S, Santalucia P, Argano C, REPOSI Investigators et al (2014) Gender-differences in disease distribution and outcome in hospitalized elderly: data from the REPOSI study. Eur J Intern Med 25(7):617-623

20. Zewari S, Vos P, van den Elshout F et al (2017) Obesity in COPD: revealed and Unrevealed Issues. COPD 14(6):663-673

21. Schnell K, Weiss CO, Lee T et al (2012) The prevalence of clinically-relevant comorbid conditions in patients with physician-diagnosed COPD: a cross-sectional study using data from NHANES 1999-2008. BMC Pulm Med. 12:26

22. Schane RE, Walter LC, Dinno A et al (2008) Prevalence and risk factors for depressive symptoms in persons with chronic obstructive pulmonary disease. J Gen Intern Med 23(11):1757-1762

23. Dodd JW, Charlton RA, van den Broek MD et al (2013) Cognitive dysfunction in patients hospitalized with acute exacerbation of COPD. Chest 144(1):119-127

24. Yohannes AM, Chen W, Moga AM et al (2017) Cognitive impairment in chronic obstructive pulmonary disease and chronic heart failure: a systematic review and meta-analysis of observational studies. J Am Med Dir Assoc. 18(5):e1-451

25. Hanania NA, Sharma G, Sharafkhaneh A (2010) COPD in the elderly patient. Semin Respir Crit Care Med 31(5):596-606
26. Fried LP, Bandeen-Roche K, Kasper JD et al (1999) Association of comorbidity with disability in older women: the Women's Health and Aging Study. J Clin Epidemiol 52(1):27-37

27. García-Pérez L, Linertová R, Lorenzo-Riera A et al (2011) Risk factors for hospital readmissions in elderly patients: a systematic review. QJM 104:639-651

28. Corrao S, Natoli G, Nobili A, RePoSI Investigators et al (2019) Comorbidity does not mean clinical complexity: evidence from the RePoSI register. Intern Emerg Med. https://doi.org/10.1007/ s11739-019-02211-3

29. Ozyilmaz E, Kokturk N, Teksut G et al (2013) Unsuspected risk factors of frequent exacerbations requiring hospital admission in chronic obstructive pulmonary disease. Int J Clin Pract 67(7):691-697

30. Ershler WB, Chen K, Reyes EB et al (2005) Economic burden of patients with anemia in selected diseases. Value Health. 8(6):629-638

31. Cote C, Zilberberg MD, Mody SH et al (2007) Haemoglobin level and its clinical impact in a cohort of patients with COPD. Eur Respir J 29(5):923-929

32. Weiss G, Goodnough LT (2005) Anemia of chronic disease. N Engl J Med 352(10):1011-1023

33. Corrao S, Argano C, Natoli G, REPOSI Investigators et al (2018) Disability, and not diabetes, is a strong predictor of mortality in oldest old patients hospitalized with pneumonia. Eur J Intern Med. 54:53-59

34. Lanièce I, Couturier P, Dramé $M$ et al (2008) Incidence and main factors associated with early unplanned hospital readmission among French medical inpatients aged 75 and over admitted through emergency units. Age Ageing 37(4):416-422

35. Lohman MC, Scherer EA, Whiteman KL et al (2018) Factors Associated With Accelerated Hospitalization and Re-hospitalization Among Medicare Home Health Patients. J Gerontol A Biol Sci Med Sci 73(9):1280-1286

36. Wang Y, Stavem K, Dahl FA et al (2014) Factors associated with a prolonged length of stay after acute exacerbation of chronic obstructive pulmonary disease (AECOPD). Int J Chron Obstruct Pulmon Dis. 9:99-105

37. Barabási AL, Gulbahce N, Loscalzo J (2011) Network medicine: a network-based approach to human disease. Nat Rev Genet 12(1):56-68

38. Fabbri LM, Rabe KF (2007) From COPD to chronic systemic inflammatory syndrome? Lancet 370(9589):797-799

39. Franceschi C, Campisi J (2014) Chronic inflammation (inflammaging) and its potential contribution to age-associated diseases. J Gerontol A Biol Sci Med Sci. Suppl 1:S4-S9

40. Miller J, Edwards LD, Agustí A, Evaluation of COPD Longitudinally to Identify Predictive Surrogate Endpoints (ECLIPSE) Investigators et al (2013) Comorbidity, systemic inflammation and outcomes in the ECLIPSE cohort. Respir Med 107(9):1376-1384

41. Thomsen M, Dahl M, Lange P et al (2012) Inflammatory biomarkers and comorbidities in chronic obstructive pulmonary disease. Am J Respir Crit Care Med 186(10):982-988

42. Agustí A, Edwards LD, Rennard SI, Evaluation of COPD Longitudinally to Identify Predictive Surrogate Endpoints (ECLIPSE) Investigators et al (2012) Persistent systemic inflammation is associated with poor clinical outcomes in COPD: a novel phenotype. PLoS One 7(5):e37483

43. Vanfleteren LE, Spruit MA, Groenen M et al (2013) Clusters of Comorbidities based on validated objective measurements and systemic inflammation in patients with chronic obstructive pulmonary disease. Am J Respir Crit Care Med 187(7):728-735

44. Kim YR, Hwang IC, Lee YJ et al (2018) Stroke risk among patients with chronic obstructive pulmonary disease: a systematic review and meta-analysis. Clinics. 73:e177 
45. Baty F, Putora PM, Isenring B et al (2013) Comorbidities and burden of COPD: a population-based case-control study. PLoS ONE 8(5):e63285

46. Sundh J, Ställberg B, Lisspers K et al (2011) Co-morbidity, body mass index and quality of life in COPD using the Clinical COPD Questionnaire. COPD 8(3):173-181

47. Mannino DM, Higuchi K, Yu TC et al (2015) Economic burden of COPD in the presence of comorbidities. Chest 148:138-150

48. Horita N, Goto A, Shibata Y et al (2017) Long-acting muscarinic antagonist (LAMA) plus long-acting beta-agonist (LABA) versus LABA plus inhaled corticosteroid (ICS) for stable chronic obstructive pulmonary disease (COPD). Cochrane Database Syst Rev 2:CD012066

49. Ernst P, Saad N, Suissa $S$ (2015) Inhaled corticosteroids in COPD: the clinical evidence. Eur Respir J 45(2):525-537

50. Simonetti AF, Viasus D, Garcia-Vidal C et al (2014) Management of community-acquired pneumonia in older adults. Ther Adv Infect Dis 2(1):3-16

51. Vitacca M, Paneroni M, Baiardi P et al (2016) Development of a Barthel Index based on dyspnea for patients with respiratory diseases. International Journal of Chronic Obstructive Pulmonary Disease. 11:1199-1206

52. Formiga F, Ferrer A, Padros G et al (2016) Evidence of functional declining and global comorbidity measured at baseline proved to be the strongest predictors for long-term death in elderly community residents aged 85 years: a 5-year follow-up evaluation, the OCTABAIX study. Clin Interv Aging 11:437-444
53. Singanayagam A, Schembri S, Chalmers JD (2013) Predictors of mortality in hospitalized adults with acute exacerbation of chronic obstructive pulmonary disease. Ann Am Thorac Soc 10(2):81-89

54. Mapel D (2014) Renal and hepatobiliary dysfunction in chronic obstructive pulmonary disease. Curr Opin Pulm Med 20(2):186-193

55. Satish S, Freeman DH Jr, Ray L, Goodwin JS (2001) The relationship between blood pressure and mortality in the oldest old. J Am Geriatr Soc 49(4):367-374

56. Odden MC, Peralta CA, Haan MN et al (2012) Rethinking the association of high blood pressure with mortality in elderly adults: the impact of frailty. Arch Intern Med 172:1162-1168

57. Zhang XE, Cheng B, Wang Q (2016) Relationship between high blood pressure and cardiovascular outcomes in elderly frail patients: a systematic review and meta-analysis. Geriatr Nurs. 37(5):385-392

58. Post Hospers G, Smulders YM, Maier AB, Deeg DJ, Muller M (2015) Relation between blood pressure and mortality risk in an older population: role of chronological and biological age. J Intern Med 277(4):488-497

59. Corrao S, Argano C, Natoli G, REPOSI Investigators et al (2019) Sex-differences in the pattern of comorbidities, functional independence, and mortality in elderly inpatients: evidence from the RePoSI Register. J Clin Med 8(1):81

Publisher's Note Springer Nature remains neutral with regard to jurisdictional claims in published maps and institutional affiliations. 